\title{
PENETRATION FRACTURE OF SEA ICE PLATE
}

\author{
ZDENĚK P. BAŽANT and YUAN N. LI \\ Department of Civil Engineering, Northwestern University, Evanston, IL 60208, U.S.A.
}

(Received 17 June 1994)

\begin{abstract}
The penetration fracture of sea ice plate floating on sea water is a complex fracture phenomenon. The paper analyses the basic aspects of fracture mechanics of the penetration problem, reviews in detail several recent solutions and presents a new improved method of solution. The basic relation between the applied load and the length of radial cracks, the mechanism that determines the number of radial cracks, and the size effect of the penetration load are discussed. Finally, a new fracture mechanics model for the ice plate penetration problem that takes into account part-through radial cracks is proposed.
\end{abstract}

\section{INTRODUCTION}

The problem of load capacity of floating ice plates is an important problem for various human activities in the Arctic Ocean. When an increasing upward or downward load is applied over a small area of an ice plate floating on sea water, diffuse cracks first emerge at the opposite side of the ice plate. Subsequently, several bending radial cracks develop and propagate with increasing load. The radial cracks are always closed on top of the plate if the load is applied downward. The ice plate fails when circumferential cracks are formed in the wedges between the radial cracks.

In early studies of the penetration problem, the load capacity of a floating ice plate was determined by the tensile strength criterion [e.g. Bernstein (1929)]. Nevel (1958) studied the strength of the ice plate assuming that the number of radial bending cracks is very large and that the ice plate is decomposed into wedges of a very small angle, which can be treated as beams of variable cross section. An excellent review of the early studies of the load capacity of floating ice plates was given by Kerr (1975).

Recently, fracture mechanics has been applied to solve the penetration problem. There are two reasons for introducing fracture mechanics; one reason is that the ice plate does not fail until extensive radial cracks develop, whereas the other is that there is a size effect on the nominal penetration stress (total applied force divided by the square of plate thickness). Without fracture mechanics, our knowledge of the size effect would be inadequate.

To accurately account for the effect of the radial bending cracks, linear elastic fracture mechanics (LEFM) was introduced by Bažant and Li (1994) to study the relation between the applied load and the length of radial cracks. Later, Li and Bažant (1994) studied the problem of how to determine the number of radial cracks. In these studies, which are reviewed in the first part of this paper, the radial cracks were assumed to be fully open, and interaction between the neighboring wedges across the part-through cracks was neglected. Resistance to horizontal expansion due to bending cracks causes the cracks to open only through part of the thickness of the plate, which was observed by Frankenstein (1963). This expansion induces compressive forces in the plate and thus engenders a dome effect, which plays an important role in helping to carry the vertical load.

A plate with part-through cracks is actually a three-dimensional problem. However, based on the simplifying idea of an embedded line spring, proposed by Rice and Levy (1972) in a study of plate fracture, the problem can be reduced to a two-dimensional one. Since the depth of radial bending crack opening is unknown in advance, the compliances of the line springs are unknown functions and have to be solved together with the plate problem. A set of integral equations based on the compliance influence functions will be proposed in this paper. 


\section{SCALING LAW FOR SEA ICE PLATE WITH LEFM THROUGH-CRACKS}

Consider an infinitely extending elastic plate of thickness $h$ floating on water of specific weight $\rho$. The water acts exactly as an elastic foundation of Winkler type. The differential equation of equilibrium of the plate in rectangular coordinates $x, y$ may be written as

$$
D\left(\frac{\partial^{2}}{\partial x^{2}}+\frac{\partial^{2}}{\partial y^{2}}\right)\left(\frac{\partial^{2} w}{\partial x^{2}}+\frac{\partial^{2} w}{\partial y^{2}}\right)+\rho w=0,
$$

in which $D=E h^{3} / 12\left(1-v^{2}\right)=$ cylindrical stiffness of the elastic plate of thickness $h$, $v=$ Poisson's ratio and $E=$ Young's modulus. It is convenient to introduce the length constant for the plate as $L=[D / \rho]^{1 / 4}$. $L$ may be called the flexural wavelength and it also represents the length over which an end disturbance in a semi-infinite plate decays to $\mathrm{e}^{-L}$ of the end value. The solution of the displacement $w$ caused by vertical load $P$ can be written as

$$
w=\frac{P L^{2}}{D} F(X, Y ; \alpha)
$$

in which $F(X, Y ; \alpha)$ is a non-dimensional function, $X=x / L$ and $Y=y / L$ are non-dimensional coordinates and $\alpha=a / L$ is the non-dimensional crack length. For the load-line displacement (or more generally, the corresponding conjugate generalized displacement, if the load is distributed), we have (Bažant and Cedolin, 1991)

$$
w_{0}=\frac{P L^{2}}{D} F\left(X_{0}, Y_{0} ; \alpha\right)=\frac{P L^{2}}{D} f(\alpha)
$$

The complementary energy of the structure under load $P$ is

$$
\Pi^{*}=\frac{1}{2} w_{0} P=\frac{P^{2} L^{2}}{2 D} f(\alpha)
$$

Energy balance during crack propagation requires that

$$
h G_{\mathrm{f}}=\frac{\partial \Pi^{*}}{\partial a}=\frac{1}{L} \frac{\partial \Pi^{*}}{\partial \alpha}=\frac{P^{2} L}{2 D} \frac{\mathrm{d} f(\alpha)}{\mathrm{d} \alpha},
$$

in which $G_{\mathrm{f}}=$ fracture energy of the material. From this we can solve the load $P$ that is necessary to cause crack propagation:

$$
P=\left(\frac{2 h D G_{\mathrm{f}}}{L}\right)^{1 / 2}\left(\frac{\mathrm{d} f(\alpha)}{\mathrm{d} \alpha}\right)^{-1 / 2}
$$

Let us define the nominal stress $\sigma_{\mathrm{N}}=P / h^{2}$ and the non-dimensional nominal stress $\tilde{\sigma}=\sigma_{\mathrm{N}} / f_{\mathrm{t}}^{\prime}=P / h^{2} f_{\mathrm{t}}^{\prime}$, where $f_{\mathrm{t}}^{\prime}=$ tensile strength of the ice, and denote $g(\alpha)=$ $1 /[\mathrm{d} f(\alpha) / \mathrm{d} \alpha]^{1 / 2}$. Provided that $\mathrm{d} f(\alpha) / \mathrm{d} \alpha>0$, we then have the result

$$
\tilde{\sigma}=\sqrt{\frac{E G_{\mathrm{f}}}{6\left(1-v^{2}\right) L f_{t}^{\prime 2}}} g(\alpha)=\sqrt{\frac{l_{0}}{6\left(1-v^{2}\right) L}} g(\alpha),
$$

in which $l_{0}=E G_{\mathrm{f}} / f_{\mathrm{t}}^{\prime 2}=$ Irwin's characteristic length of the process zone, representing a material length characteristic.

The most interesting consequence of (7) is that, for geometrically similar cracks (same $\alpha$, but various thicknesses $h$ ), the non-dimensional nominal strength decreases as $L^{-1 / 2}$. 
This resembles the classical scaling law of linear elastic fracture mechanics. Since $L$ is proportional to $h^{3 / 4}$, the nominal stress decreases as $h^{-3 / 8}$. The fact that the size effect is of the type $h^{-3 / 8}$ has already been shown by Slepyan (1990) and Bažant (1992). It is important to note that in terms of $L$, the size effect acquires its familiar form of $L^{-1 / 2}$ (this was not pointed out in Slepyan's work), so the general scaling law of linear fracture mechanics is preserved. It is also interesting that, as Bažant (1992) proved, the size effect $h^{-3 / 8}$ applies also to thermal fracture in floating plates caused by a critical temperature drop.

\section{PENETRATION LOAD OF SEA ICE PLATES}

When a load is gradually applied on top of a sea ice plate, radial bending cracks will grow from the center of the loading. We now assume the crack to cut across the whole plate thickness, all the way to the crack front. The relation between the penetration load and the length of radial bending cracks obeys eqn (6) [or eqn (7) in its non-dimensional form]. However, the ice plate does not fail because of radial crack propagation. Due to these radial cracks, the ice plate is decomposed into wedges (Fig. 1). The maximum applied load is reached when these wedges break by circumferential cracks due to bending, and this failure is controlled by the tensile strength of the ice. As a result, the $h^{-3 / 8}$ law does not apply to the nominal failure stress of the penetration load.

The elastic solution for the ice plate with $n$ radial cracks can be written in the form

$$
w=\frac{P L^{2}}{D} F(r, \theta ; \alpha, n)
$$

in which $n=$ number of the radial cracks (with uniform angular distribution). The compliance function $f$ is related to $F$ in a way that depends upon the distribution of the applied load. The applied load $P$ required to maintain $n$ radial cracks can be determined by eqn (6) with $h$ replaced by $n h$.

Since function $f$ approaches a constant for a large crack length $a$, the applied load can exceed any finite value for long enough radial cracks. Before this can happen, however, the wedges would break by circumferential cracks, due to their limited tensile strength. According to the plate theory, the circumferential tensile stress at the top of the wedge is

$$
\sigma=-\frac{6 M_{\theta}}{h^{2}}=\frac{6 D}{h^{2}}\left[\frac{1}{r^{2}} \frac{\partial^{2} w}{\partial \theta^{2}}+\frac{1}{r} \frac{\partial w}{\partial r}+v \frac{\partial^{2} w}{\partial r^{2}}\right]=\frac{6 P}{h^{2}} F_{\mathrm{m}}(r, \theta ; \alpha, n),
$$

where the dimensionless function $F_{\mathrm{m}}$ is related to $F$. With the notation

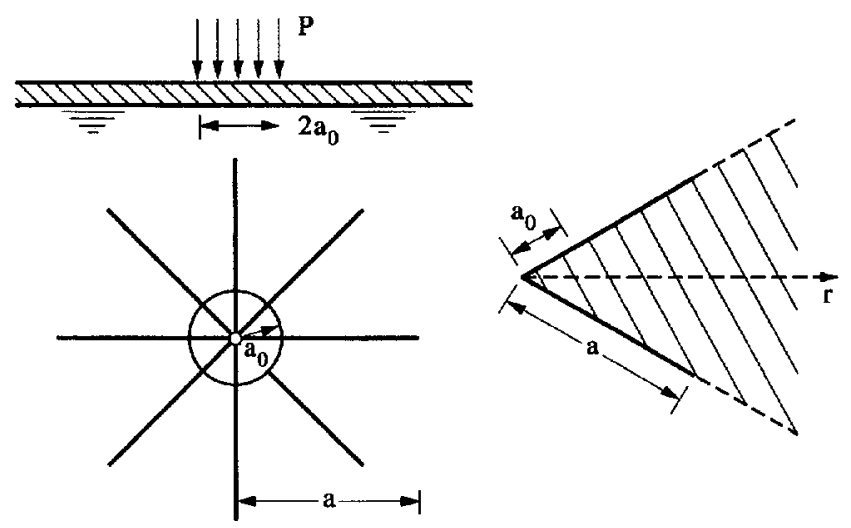

Fig. 1. Floating ice sheet subjected to vertical load and wedge geometry. 


$$
F_{\mathrm{m}}(\alpha, n)=\max _{r, \theta}\left\{\frac{\partial^{2} F(r, \theta ; \alpha, n)}{\partial x^{2}}\right\}
$$

the failure criterion can be expressed as

$$
\tilde{\sigma}_{\max }=\frac{6 P}{f_{\mathrm{t}} h^{2}} F_{\mathrm{m}}(\alpha, n) \leqslant 1
$$

Since the applied load is related to the length of radial crack through eqn (6), one obtains the following inequality:

$$
\sqrt{\frac{6 n l_{0}}{\left(1-v^{2}\right) L}} g(\alpha, n) F_{\mathrm{m}}(\alpha, n) \leqslant 1 .
$$

This inequality must be satisfied during the process of radial crack propagation, and the wedges will break if the equality holds. This yields an equation for determining the nondimensional crack length at which the wedges break at the location of maximum moment. After the crack length is determined, the applied load can be found from eqn (6).

The general approach described in this section was used by Bažant and Li (1994) to obtain numerical solutions. However, the present formulation embraces a broader range of fracture phenomena in sea ice plates.

\section{THEORY OF INITIAL CRACK SPACING}

As noticed by Frankenstein (1963), the number of radial cracks formed in a penetration test is a highly variable quantity. However, for different sizes of the loading device, his tests demonstrated that, despite random scatter, there is a deterministic trend. The average number of radial cracks depends on the diameter of the penetrator.

As is well known, LEFM cannot directly model the crack initiation, since when the crack length is zero, the energy release rate is also zero. The Griffith's equation of energy balance cannot be applied. Therefore, the strength concept must be used in some form. When the load is uniformly distributed over a circular area of radius $\alpha_{0}$, the plate equation (1) can be solved analytically to determine the load $P_{\mathrm{f}}$ at which the cracks first occur (i.e. $f_{\mathrm{t}}$ is reached) :

$$
P_{\mathrm{f}}=\frac{\pi \alpha_{0}}{3(1+v) \operatorname{kei}^{\prime}\left(\alpha_{0}\right)} f_{\mathrm{t}} h^{2}=\frac{f_{\mathrm{t}} h^{2}}{C\left(\alpha_{0}\right)},
$$

where kei is a Kelvin function [see Bernstein (1929), Wyman (1950)].

When eqn (13) is satisfied, cracks start to form in the ice. However, the strength theory alone cannot determine the number of radial cracks, and the energy criterion must be employed. The stress intensity factor is an increasing function initially and later turns into a decreasing function. In other words, the crack propagation is unstable (or dynamic) initially, but becomes stable when $\alpha$ is large enough. Therefore, once the radial cracks start growing, they will not stop until the energy release rate starts to decrease and the energy balance equation (6) becomes satisfied with the load remaining at the level of $P_{\mathrm{f}}$. Such an equation can be written in a non-dimensional form as

$$
\frac{n}{f_{x}\left(\alpha_{0}, \alpha, n\right)}=\frac{L}{l_{0}} \frac{3\left(1-v^{2}\right)}{\pi C^{2}\left(\alpha_{0}\right)}
$$

For each given $n$ (and $\alpha_{0}$ as well, of course), eqn (14) makes it possible to determine the initial length of radial cracks, which will henceforth be denoted as $\alpha_{t}$. However, $\alpha_{t}$ is generally different for different $n$. To determine $n$ we need one more condition. The 


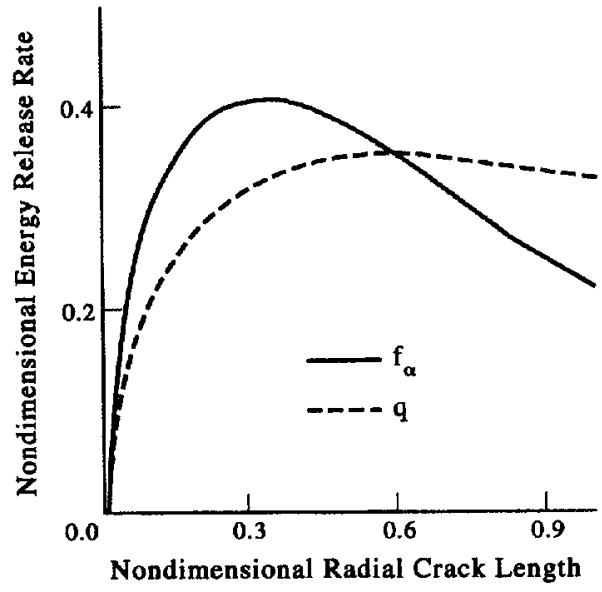

Fig. 2. General relation between average energy release rate function $q$ and the energy release rate function $f_{\alpha}$.

additional condition can be obtained by considering the finite energy released by the sudden dynamic crack length jump from zero to $\alpha_{t}$, which is accompanied by an increase of deflection under the constant load. The energy released is assumed to be entirely consumed by creating the crack surfaces, i.e. no other dissipation of energy exists. So, $\left[f\left(\alpha_{0}, \alpha_{1}, n\right)-f\left(\alpha_{0}, 0, n\right)\right] P_{\mathrm{f}}^{2} L^{2} / 4 \pi D=n L \alpha_{\mathrm{t}} h G_{\mathrm{f}}$, or in non-dimensional form :

$$
\frac{n}{q\left(\alpha_{0}, \alpha_{\mathrm{t}}, n\right)} \doteq \frac{\alpha_{\mathrm{t}} n}{f\left(\alpha_{0}, \alpha_{\mathrm{t}}, n\right)-f\left(\alpha_{0}, 0, n\right)}=\frac{3\left(1-v^{2}\right)}{\pi C^{2}\left(\alpha_{0}\right)} \frac{L}{l_{0}}
$$

The average energy release equation (15) and the energy release equation (14) can be simultaneously satisfied if, and only if, the function $q$ is equal to $f_{\alpha}$, which occurs when $q$ reaches its maximum (Fig. 2). There is only one root in the entire range. The maximum of $q$ is always smaller than the maximum of $f_{\alpha}$. Denoting the maximum value of $q\left(\alpha_{0}, \alpha, n\right)$ as $Q\left(\alpha_{0}, n\right)$, which is listed in Table 1 , we can rewrite the energy release rate equation (14) as

$$
\frac{n}{Q\left(\alpha_{0}, n\right)}=\frac{L}{l_{0}} \frac{3\left(1-v^{2}\right)}{\pi C^{2}\left(\alpha_{0}\right)}
$$

Experiments (Frankenstein, 1963) showed that $n$ increases with increasing punch size, and this trend is indeed captured by eqn (16): because $\alpha_{0}$ increases with punch size, whereas $C\left(\alpha_{0}\right)$ decreases with $\alpha_{0}$, the final result is an increase in the right-hand side of the equation, and thus an increase in $n$. The predicted number of radial cracks is quite close to the field observations under proper assumption of material properties of the ice plates.

Note that the smallest value of the right-hand side of eqn (16) is zero, while the lefthand side has a minimum, denoted as $U\left(\alpha_{0}\right)$, which is larger than zero, and occurs at $n=3$. Then, through eqn (16), one can define the threshold length $L_{0}$ as

Table 1. Function $Q$ and $n / \mathrm{Q}$

\begin{tabular}{ccccccccccc}
\hline$\alpha_{0}$ & $n$ & 2 & 3 & 4 & 5 & 6 & 8 & 12 & 24 & 36 \\
\hline \multirow{2}{*}{0.1} & $Q$ & 0.358 & 0.580 & 0.751 & 0.880 & 0.978 & 1.113 & 1.256 & 1.397 & 1.433 \\
& $n / \mathrm{Q}$ & 5.585 & 5.175 & 5.325 & 5.682 & 6.135 & 7.186 & 9.553 & 17.18 & 25.12 \\
0.01 & $Q$ & 0.339 & 0.569 & 0.741 & 0.867 & 0.962 & 1.091 & 1.223 & 1.348 & 1.379 \\
& $n / \mathrm{Q}$ & 5.893 & 5.271 & 5.400 & 5.767 & 6.236 & 7.333 & 9.732 & 17.80 & 26.11 \\
\hline
\end{tabular}




$$
L_{0}=\frac{\pi l_{0}}{3\left(1-v^{2}\right)} C^{2}\left(\alpha_{0}\right) U\left(\alpha_{0}\right) \text {. }
$$

When the action length $L$ is smaller than $L_{0}$, no radial cracks form during loading, and the only way an ice plate may fail is by a conic crack under the load. For instance, for an ice sheet with $l_{0}=0.2 \mathrm{~m}$ and $\alpha_{0}=0.1, L_{0}=3.91 \mathrm{~m}$. When $h$ is measured in meters, the action distance $L$ can be related to $h$ as $L \approx 16 h^{3 / 4}$; thus $h_{0} \approx 0.15 \mathrm{~m}$. A more detailed discussion of the analysis presented in this section is given by Li and Bažant (1994).

\section{SIZE EFFECT ON THE MAXIMUM PENETRATION LOAD}

The size effect on non-dimensional nominal stress $\sigma_{\mathrm{N}}\left(=P_{\max } / h^{2} f_{1}\right)$ depends on how the similarity is defined. If it is defined so that $\alpha_{0}$ be constant for varying $h$, then there is no size effect if the effect of bending modulus and of changing crack number $n$ are neglected, as is shown in Fig. 3(a). In the calculation it is assumed that $l_{0}=0.2 \mathrm{~m}$ and $L=16 h^{3 / 4}$. The size effect, due to a change in $n$ under constant $\alpha_{0}$, is plotted in Fig. 3(b). The left end of the curves corresponds to the threshold value $L_{0}$ as discussed in the previous section.

It may be emphasized that the size effect due to a change in $n$ can be seen only through a two-dimensional analysis. The preceding one-dimensional analysis by Bažant and $\mathrm{Li}$ (1994), in which the wedges were treated as very narrow beams, could not reflect such a size effect because in the narrow wedge beams the maximum bending moment is independent of the wedge angle, and thus independent of $n$.

When the ratio $a_{0} / h$ is kept constant for various $h$, a reversed size effect is superimposed on the size effect mentioned above. Since $\alpha_{0}=\left(a_{0} / h\right)\left[h\left(1-v^{2}\right) / E\right]^{1 / 4}, \alpha_{0}$ actually increases with $h$, and we know that when $\alpha_{0}$ increases, the maximum value of the nominal stress increases. Thus the size effect gets reversed; the larger $h$ is, the larger $\sigma_{\mathrm{N}}$ is. However, since the dependence of $x_{0}$ on $h$ is weak in the normal range of thickness, such a reversed size effect can often be neglected.

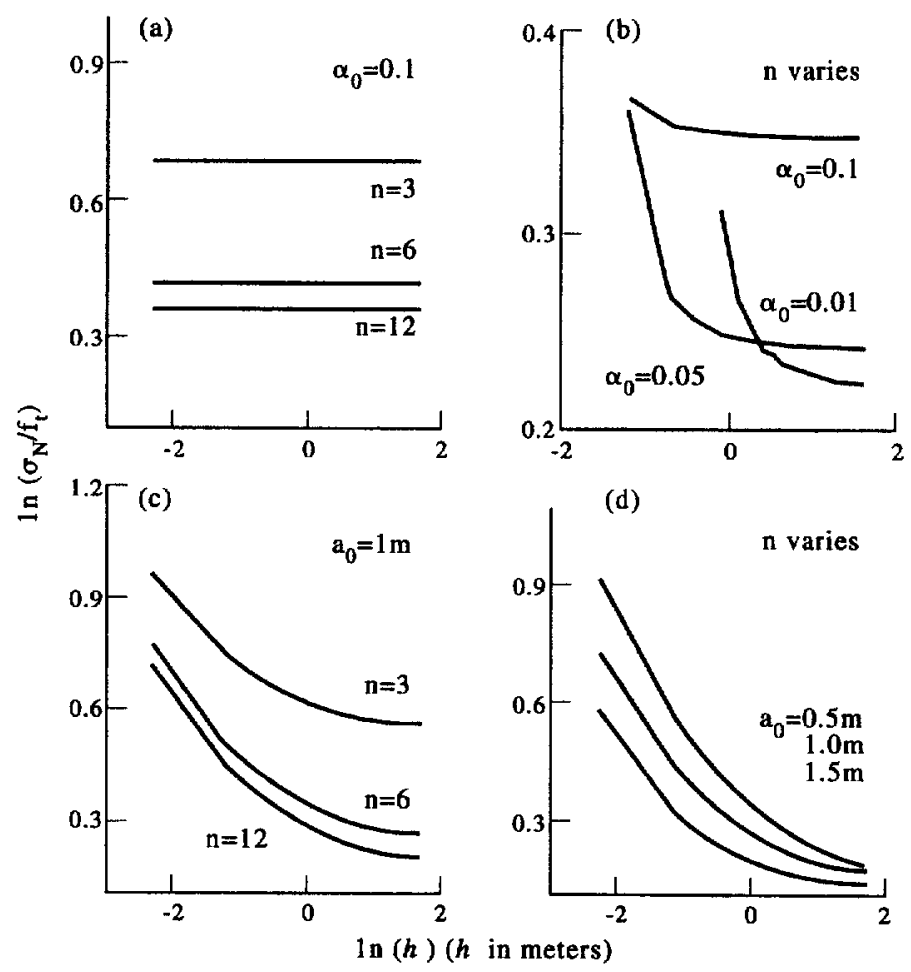

Fig. 3. Size effect for (a) constant $\alpha_{0}$ and constant $n$; (b) constant $\alpha_{0}$ and varying $n$; (c) constant $a_{0}$ and constant $n ;$ (d) constant $a_{0}$ and varying $n$. 
Actually, keeping $a_{0}$ constant for all $h$ is the case that is more relevant to the practical problem, in which an aircraft of a fixed and known contact area wants to land safely on the ice plate, or a submarine of a fixed and known contact area of its sail wants to penetrate upward through the ice. For this case, $\alpha_{0}$ decreases with $h$, and so the maximum nominal stress decreases with $h$, too, as shown in Fig. 3(c) for several chosen $n$. This type of size effect has already been discussed by Bažant and $\mathrm{Li}$ (1994). It is interesting to note that even with constant $a_{0}, n$ still increases with $h$, because the rate of increase of $L$ is faster than that of function $C^{2}$. Thus the overall effect of changing $n$ is to enhance the size effect under the condition of constant $a_{0}$, as can be seen from Fig. 3(d).

\section{PENETRATION LOAD OF THE ICE PLATE WITH PART-THROUGH RADIAL CRACKS}

In the preceding analysis, it is assumed that the surfaces of radial cracks are free from stress. In reality, the radial cracks are only partially open. If the load is applied on top of the ice plate, the top portion of the plate near the load is under compression. In the field, radial cracks manifest themselves as a whitening on the top surface of the ice (Frankenstein, 1963), and they usually become apparent only after the load is removed. In other words, the radial cracks only open partially. If the downward load is applied on the top surface, then the radial cracks are opened only in the lower part of the plate while the upper surfaces remain closed due to the compression generated by the applied load. This tends to produce in-plane compression forces in the plate, whose resultant is shifted above the mid-thickness. Thus a dome effect develops and helps to carry the load. The previous analysis ignored the dome effect.

The partial opening of the radial cracks is a three-dimensional phenomenon. A detailed three-dimensional fracture mechanics analysis is computationally expensive, if not intractable. In the present analysis, it is proposed to model the partial crack as a line spring in the crack line. In this approach, the plate with radial cracks, floating on water, is still analysed as a two-dimensional problem floating on water.

The difference from our previous analysis lies in how the radial cracks are treated. Since the cracking is only partial, the surfaces of radial cracks may be imagined to be connected with line springs. The compliances of these springs are such that they match the increase of the compliances due to a partial crack of the same depth in an infinite strip subjected to remote tension and bending in the direction normal to the crack.

The idea of incorporating the compliance increase due to the presence of a partthrough crack was proposed by Okamura et al. (1972). Rice and Levy (1972) used the same idea to solve the stress intensity factors of a partially cracked plate subjected to in-plane and out-of-plane loads. In their paper, they stated various assumptions involved in such an approach to solve part-through surface cracks of given depth profile. Our problem, however, is different. The depth profile of the radial cracks is not known in advance. Determination of the radial crack profile is part of our problem.

Denote $\Delta$, the crack expansion (in the circumferential direction) and $\theta$, the crack surface rotation (about the radial ray); $\theta$ and $\Delta$ vary with radial distance $r$ and the variation is unknown in advance. Denote by $N$ and $M$ the normal force (force per unit length) and bending moment (moment per unit length) associated with $\Delta$ and $\theta$ (Fig. 4). A positive moment is that which causes the tension on the bottom surface of the plate, and a tensile normal force is taken as positive. Furthermore, denote by $b(r)$ the depth of the radial cracks at position $r$. Since we have replaced the partially cracked surfaces with line springs, the surface forces and the surface displacements can be related as

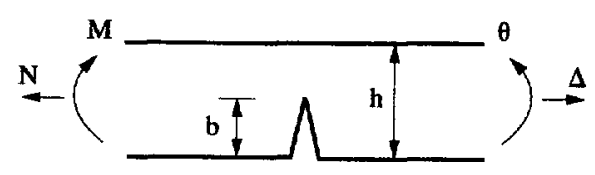

Fig. 4. Definitions for $N, M, \Delta$ and $\theta$. 


$$
\Delta=\lambda_{11} N+\lambda_{12} M, \quad \theta=\lambda_{21} N+\lambda_{22} M \text {. }
$$

The compliances $\lambda_{i j},(i, j=1,2)$ of the line springs can be written in terms of stress intensity factors (SIF) $k_{i}(i=1,2)$, where $k_{1}=$ SIF in an infinite strip of width $h$ with a single sided notch of depth $b$ loaded remotely by a unit $N$, and $k_{2}=$ SIF of the same strip by a unit $M$ :

$$
\lambda_{i j}=2 \frac{1-v^{2}}{E} \int_{0}^{b} k_{i}(z) k_{j}(z) \mathrm{d} z, \quad i, j=1,2
$$

Approximate expressions for $\lambda_{11}$ and $\lambda_{22}$ are given by Tada et al. (1985), and so only the empirical formula for $\lambda_{12}$ needs to be calibrated through eqn (19).

Rotation $\theta$ and expansion $\Delta$ are related to the elastic solution of the ice plate (which has $n$ radial cracks of length $a$ ) as follows :

$$
\begin{gathered}
\theta(r)=C_{M P}(r) \frac{P}{n}-\int_{0}^{a} C_{M M}\left(r, r^{\prime}\right) M\left(r^{\prime}\right) \mathrm{d} r \\
\Delta(r)=-\int_{0}^{a} C_{N N}\left(r, r^{\prime}\right) N\left(r^{\prime}\right) \mathrm{d} r^{\prime}
\end{gathered}
$$

where $C_{M P}(r)=$ rotation of the plate at $r$ due to a unit $P, C_{M M}\left(r, r^{\prime}\right)$ is the rotation of the plate at $r$ due to a unit moment acting on the crack surfaces at $r^{\prime}$, and $C_{N N}\left(r, r^{\prime}\right)$ is the crack expansion at $r$ due to a unit normal force $N$ at $r^{\prime}$. The negative sign in front of the integrals is due to the fact that positive forces on the crack surfaces cause the crack to close. All these compliance influence functions can be solved by numerical means, such as the finite element method or the finite difference method.

Substituting $\theta$ and $\Delta$ in eqn (18), one obtains the following integral equations :

$$
\begin{gathered}
\lambda_{11} N(r)+\lambda_{12} M(r)=-\int_{0}^{a} C_{N N}\left(r, r^{\prime}\right) N\left(r^{\prime}\right) \mathrm{d} r^{\prime} \\
\lambda_{21} N(r)+\lambda_{22} M(r)=C_{M P}(r) \frac{P}{n}-\int_{0}^{a} C_{M M}\left(r, r^{\prime}\right) M\left(r^{\prime}\right) \mathrm{d} r^{\prime} .
\end{gathered}
$$

These equations can be used to solve for the unknown functions $N$ and $M$ along the radial crack surfaces if the radial crack depth, as well as the applied load $P$, are known. However, the radial crack profile as well as the applied load are yet to be determined.

To close the equation, we postulate that at any point of the radial cracks, the total stress intensity factor at the crack tip be equal to $K_{\mathrm{f}}$, the fracture toughness of the ice. That is

$$
k_{1}[b(r)] N(r)+k_{2}[b(r)] M(r)=K_{\mathrm{f}} \quad 0 \leqslant r \leqslant a .
$$

This equation can be used to determine the depth profile of radial cracks if the forces $M$ and $N$ are known. The fracture energy $G_{\mathrm{f}}$ is related to the fracture toughness $K_{\mathrm{f}}$ by the equation $G_{\mathrm{f}}=K_{\mathrm{f}}^{2}\left(1-v^{2}\right) / E$. In order to derive an equation to determine the applied load, let us introduce the complementary energy of the ice plate with $n$ radial cracks. The basic unknowns are $N(r), M(r), b(r)$ and $a$, and the parameter is load $P$. So, the energy potential for the problem may be written as: 


$$
\begin{aligned}
\Pi_{c}=\frac{1}{2} \int_{0}^{a} \int_{0}^{a} C_{M M}\left(r, r^{\prime}\right) & M(r) M\left(r^{\prime}\right) \mathrm{d} r \mathrm{~d} r^{\prime}-\frac{P}{n} \int_{0}^{a} C_{M P}(r) M(r) \mathrm{d} r \\
& +\frac{P^{2}}{2 n^{2}} C_{P P}+\frac{1}{2} \int_{0}^{a} \int_{0}^{a} C_{N N}\left(r, r^{\prime}\right) N(r) N\left(r^{\prime}\right) \mathrm{d} r \mathrm{~d} r^{\prime} \\
& +\frac{1}{2} \int_{0}^{a}\left[\lambda_{11} N^{2}(r)+2 \lambda_{12} N(r) M(r)+\lambda_{22} M^{2}(r)\right] \mathrm{d} r-G_{\mathrm{f}} \int_{0}^{a} b(r) \mathrm{d} r .
\end{aligned}
$$

It can be easily verified that eqns (22) and (23) are equivalent to the condition that the first variation of $\Pi_{c}$ with respect to $N$ and $M$ be zero. Equation (24) is equivalent to the condition that the first variation of $\Pi_{c}$ with respect to $b(r)$ be equal to zero, that is

$$
\delta_{b} \Pi_{c}=\frac{1-v^{2}}{E} \int_{0}^{a}\left[k_{1} N(r)+k_{2} M(r)\right]^{2} \delta b(r) \mathrm{d} r-\int_{0}^{a} G_{\mathrm{f}} \delta b(r) \mathrm{d} r=0 .
$$

Since the elastic compliance functions $C$ are defined on plates with stress-free radial cracks, they are independent of the crack depth profile $b(r)$. The applied load can be determined by the condition that the variation of $\Pi_{c}$ with respect to $a$ be equal to zero

$$
\begin{aligned}
\frac{1}{2} \int_{0}^{a} \int_{0}^{a} \frac{\partial C_{M M}\left(r, r^{\prime}\right)}{\partial a} M(r) M\left(r^{\prime}\right) \mathrm{d} r \mathrm{~d} r^{\prime}-\frac{P}{n} \int_{0}^{a} & \frac{\partial C_{M P}(r)}{\partial a} M(r) \mathrm{d} r+\frac{P^{2}}{2 n^{2}} \frac{\partial C_{P P}}{\partial a} \\
& +\frac{1}{2} \int_{0}^{a} \int_{0}^{a} \frac{\partial C_{N N}\left(r, r^{\prime}\right)}{\partial a} N(r) N\left(r^{\prime}\right) \mathrm{d} r^{\prime} \mathrm{d} r^{\prime}=0
\end{aligned}
$$

In calculating the variation, it is assumed that $b(r)$ is independent of the crack length $a$. If one defines $k_{N}(r), k_{M}(r)$ and $k_{P}$ as the stress intensity factor at the radial crack tip due to corresponding unit forces, then the derivative of the elastic compliance functions with respect to crack length can be related to these stress intensity factors as

$$
\begin{gathered}
\frac{\partial C_{M M}\left(r, r^{\prime}\right)}{2 h \partial a}=\frac{1-v^{2}}{E} k_{M}(r) k_{M}\left(r^{\prime}\right), \quad \frac{\partial C_{M P}(r)}{2 h \partial a}=\frac{1-v^{2}}{E} k_{M}(r) k_{P}, \quad \frac{\partial C_{P P}}{2 h \partial a}=\frac{1-v^{2}}{E} k_{P}^{2} \\
\frac{\partial C_{N N}\left(r, r^{\prime}\right)}{2 h \partial a}=\frac{1-v^{2}}{E} k_{N}(r) k_{N}\left(r^{\prime}\right) .
\end{gathered}
$$

As a result, eqn (27) can be equivalently cast into the form

$$
\left(k_{P} \frac{P}{n}-\int_{0}^{a} k_{M}(r) M(r) \mathrm{d} r\right)^{2}+\left(\int_{0}^{a} k_{N}(r) N(r) \mathrm{d} r\right)^{2}=0 .
$$

This equation can be satisfied if, and only if, the expressions in each bracket vanish separately.

Equations (22)-(24) and (30) provide the sufficient number of equations to solve the unknowns $N(r), M(r), b(r)$ and $a$. In the calculation, the crack length $a$ is treated as given data, the applied load $P$ is solved as an unknown. The system of equations needs to be solved iteratively. However, it must be cautioned that eqn (18) is only valid when the crack is opened under the remote loading. If the crack is totally closed, then the compliance increment $\lambda_{i j}$ due to the presence of the crack must be modified accordingly.

Due to the introduction of the dome effect, the relation between the applied load and the length of radial cracks, and the size effect of the nominal penetration stress will be 
changed. The theory of initial crack spacing remains the same, but the actual conditions involved in the analysis will be modified. These questions will be analysed in another paper.

\section{CONCLUSIONS}

In the traditional approach, the failure of a brittle structure is defined by the first appearance of fracture. This is the basis for various strength criteria. To understand what happens after strength criteria are violated, one needs to employ fracture mechanics. Looking into the detail of structural failure is the modern trend of solid mechanics. The penetration of floating ice plates provides a good example of how fracture investigation is conducted to understand the actual failure process.

The major points of this paper are as follows:

(1) According to linear elastic fracture mechanics, the nominal stress (load divided by the square of the thickness) required to drive geometrically similar bending cracks in the floating ice plate is proportional to (thickness) ${ }^{-3 / 8}$. However, in terms of flexural wavelength $L$, the nominal stress is proportional to $L^{-1 / 2}$.

(2) The propagation of radial bending cracks in the floating ice plate is stable. The effect of radial cracking is the decomposition of the ice plate into ice wedges.

(3) The number of radial bending cracks may be determined by considering the following three conditions: (i) the tensile stress under the applied load must be equal to the tensile strength of the ice so that cracks can develop (this yields the crack initiation load); (ii) the initial crack length must satisfy the Griffith criterion; (iii) the total work done by the load during the crack initiation must be equal to the total surface energy of the newly formed crack surface.

(4) The effect of size on the nominal penetration stress depends on many factors. Among them are the exact definition of geometric similarity and the actual number of radial cracks. Relevant to practice is a definition of similarity in which the load area is constant. There is a significant size effect on the nominal stress as a function of thickness.

(5) The penetration problem of the ice plate with part-through radial cracks can be described by the new integral equations proposed in this paper. The part-through radial cracks are replaced by ideal line springs with appropriate compliances that can be determined by linear fracture mechanics as functions of the crack depth.

Acknowledgement-Financial support under Grant N00014-91-J-1109 (monitored by Dr Y. Rajapakse) from the Office of Naval Research to Northwestern University is gratefully acknowledged.

\section{REFERENCES}

Bažant, Z. P. (1992). Large scale thermal bending fracture of sea ice plate. J. Geophys. Res.—Oceans 97 (C11), $17739-17751$

Bažant, Z. P. and Cedolin, L. (1991). Stability of Structures: Elastic, Inelastic, Fracture and Damage Theories. Oxford University Press, New York.

Bažant, Z. P. and Li, Y. N. (1994). Penetration through floating sea ice plate and size effect: simplified fracture analysis. J. Engng Mech. ASCE 120(6), 1304-1321

Bernstein, S. (1929). The Railway Ice Crossing. Trudy Nauchno-Techniches-kogo Komiteta Narodnogo Komissariata Putei, Soobshchenniia, Vol. 84 (in Russian).

Frankenstein, E. G. (1963). Load test data for lake ice sheet. Technical Report 89, U.S. Army Cold Regions Research and Engineering Laboratory, Hanover, $\mathrm{N} \mathrm{H}$.

Kerr, A. D. (1975). The bearing capacity of floating ice plates subjected to static or quasi-static loads--A critical survey. Research Report 333, U.S. Army Cold Regions Research and Engineering Laboratory, Hanover, N H.

Li, Y. N. and Bažant, Z. P. (1994). Penetration fracture of floating ice-plate : 2D analysis and size effect. J. Engng Mech. ASCE 120(7), 1481-1498.

Nevel, D. E. (1958). The theory of narrow infinite wedge on an elastic foundation. Transactions, Engineering Institute of Canada, Vol. 2, No. 3.

Okamura, H., Watanabe, K. and Takano, T. (1972). Applications of the compliance concept in fracture mechanics. In 6th National Symposium on Fracture Mechanics, Progress in Flaw Growth and Fracture Toughness Testing, ASME Special Technical Publication \# 536, pp. 423-439. 
Rice, J. R. and Levy, N. (1972). The part-through surface crack in an elastic plate. J. Appl. Mech. ASME 39, $185-194$.

Slepyan, L. I. (1990). Modeling of fracture of sheet ice. Izv. AN SSSR Mekh. Tver. Tela 25(2), 151-157.

Tada, H., Paris, P. C. and Irwin, G. R. (1985), Stress analysis of cracks handbook. Del Research Corp, Hellertown, PA.

Wyman, M. (1950). Deflections of an infinite plate. Canadian J. Res. A28. 